\title{
Fludarabine-mediated suppression of the excision repair enzyme ERCCI contributes to the cytotoxic synergy with the DNA minor groove crosslinking agent SJG-I36 (NSC 69450I) in chronic lymphocytic leukaemia cells
}

\author{
C Pepper, ${ }^{*}$, H Lowe ${ }^{2}$, C Fegan', C Thurieau ${ }^{3}$, DE Thurston ${ }^{4}$, JA Hartley ${ }^{2}$ and P Delavault ${ }^{3}$ \\ 'Department of Haematology, School of Medicine, Cardiff University, Heath Park, Cardiff, UK; ${ }^{2}$ Cancer Research UK Drug-DNA Interactions Research \\ Group, Department of Oncology, University College London, London, UK; ${ }^{3}$ psen, Research \& Development, Paris, Cedex 16, France; ${ }^{4}$ Department of \\ Pharmaceutical and Biological Chemistry, Cancer Research UK Gene Targeted Drug Design Research Group, School of Pharmacy, University of London, \\ Brunswick Square, London, UK
}

In this study, we set out to establish whether fludarabine could enhance the DNA interstrand crosslinking capacity of SJG- I36 in primary human chronic lymphocytic leukaemia (CLL) cells and thereby offer a rationale for its clinical use in combination with SJGI36. SJG-I 36 rapidly induced DNA crosslinking in primary CLL cells which was concentration-dependent. Further, the level of crosslinking correlated with sensitivity to SJG-I36-induced apoptosis $(P=0.00 \mathrm{I})$ and higher levels of crosslinking were induced by the combination of SJG-I 36 and fludarabine $(P=0.002)$. All of the samples tested $(n=40)$ demonstrated synergy between SJG- I 36 and fludarabine (mean combination index $(\mathrm{Cl})=0.54 \pm 0.2$ ) and this was even retained in samples derived from patients with fludarabine resistance (mean $\mathrm{Cl}=0.62 \pm 0.3$ ). Transcription of the excision repair enzyme, $E R C C I$, was consistently increased (20/20) in response to SJG-I36 ( $P<0.000$ I). In contrast, fludarabine suppressed ERCCI transcription $(P=0.04)$ and inhibited SJG- I 36-induced ERCCI transcription when used in combination $(P=0.00 \mathrm{I})$. Importantly, the ability of fludarabine to suppress ERCCI transcription correlated with the degree of synergy observed between SJG-I36 and fludarabine $\left(r^{2}=0.28 ; P=0.017\right)$ offering a mechanistic rationale for the synergistic interaction. The data presented here provides a clear indication that this combination of drugs may have clinical utility as salvage therapy in drug-resistant CLL.

British Journal of Cancer (2007) 97, 253-259. doi:I0.1038/sj.bjc.6603853 www.bjcancer.com

Published online 19 June 2007

(c) 2007 Cancer Research UK

Keywords: SJG-136; PBD dimer; DNA crosslinking; chronic lymphocytic leukaemia; apoptosis; synergy

B-cell chronic lymphocytic leukaemia (CLL) is an incurable malignancy with a variable clinical course (Bosch and Montserrat, 2002). Outcome for advanced-stage CLL patients is poor, with an expected median survival of 3 years (Lee et al, 1987). Although the introduction of purine nucleoside analogues (Zhu et al, 2004) and monoclonal antibody therapies (Byrd et al, 2001; Keating et al, 2002), either as single agents or in combination (Byrd et al, 2003; Kay, 2006), has led to significantly increased response rates, none of these options is curative. Consequently, there is a need to identify new agents for the treatment of CLL, particularly agents that have the potential to complement existing therapeutic options and overcome drug resistance.

SJG-136 (NSC 694501, BN2629, SG2000) is a novel DNA crosslinking agent that binds in a sequence-specific manner the minor groove of the helix (Gregson et al, 2001; Hartley et al, 2004). It is structurally novel compared to other clinically used DNA crosslinking agents and has exhibited a unique multilog differential pattern of activity in the NCI 60 cell line screen (Hartley

*Correspondence: Dr C Pepper; E-mail: peppercj@cf.ac.uk

Received 12 January 2007; revised 3 May 2007; accepted 21 May 2007; published online 19 June 2007 et al, 2004). SJG-136 also displayed potent activity against several human tumours in vivo (Alley et al, 2004), and phase I clinical trials against both solid tumours and haematological malignancies are currently underway. We have previously shown that SJG-136 is extremely cytotoxic to CLL cells in vitro, exhibiting $\mathrm{LD}_{50}$ values more than 2 logs lower than fludarabine, the current treatment of choice for this condition (Pepper et al, 2004). Furthermore, SJG136 exhibited differential cytotoxicity since it was less effective at inducing apoptosis in normal B- and T-lymphocytes derived from CLL patients.

The formation of drug-induced crosslinks between two complementary strands of DNA, interstrand crosslinking (ICL), is considered to be critical to the activity of bifunctional alkylating agents, and there is clear evidence that their formation and subsequent persistence correlates to in vitro cytotoxicity (Sunters et al, 1992). In this context, it has been previously demonstrated that fludarabine can inhibit excision and repair of ICL and by so doing contributes to the synergy observed between fludarabine and cisplatin (Yang et al, 1995; $\mathrm{Li}$ et al, 1997). This inhibition of ICL repair was associated with the inhibition of the nucleotide excision repair enzyme, ERCC1. In addition, a recent report clearly demonstrated a role for ERCC1 in the repair of SJG-136-induced DNA ICLs (Clingen et al, 2005). Therefore, 
this present study was designed to investigate whether fludarabinemediated inhibition of ERCC1-mediated DNA repair could positively impact on SJG-136 ICL patterns and enhance the resulting cytotoxicity of SJG-136 in CLL cells.

\section{MATERIALS AND METHODS}

\section{Patients' cells and clinical details}

Peripheral blood samples from 40 patients with CLL were obtained with the patients' written informed consent in accordance with the conditions for ethical approval granted by the Southeast Wales Research Ethics Committee (no. 02/4806). Chronic lymphocytic leukaemia was defined by clinical criteria, and staging was based on the Binet system (Binet et al, 1981). None of the previously treated patients had received therapy for at least 1 month before the study. $\mathrm{V}_{\mathrm{H}}$ gene mutational status, CD38 expression and ZAP-70 expression was available for all of the patients under investigation. The clinical characteristics of the patient cohort are summarised in Table 1.

\section{Primary CLL cell-culture conditions}

Freshly isolated peripheral blood lymphocytes $\left(1 \times 10^{6} \mathrm{ml}^{-1}\right)$ were cultured in RPMI medium (Invitrogen, Paisley, UK), supplemented with $100 \mathrm{U} \mathrm{ml}^{-1}$ penicillin, $100 \mu \mathrm{g} \mathrm{ml}^{-1}$ streptomycin and $10 \%$ fetal calf serum. Lymphocytes were incubated at $37^{\circ} \mathrm{C}$ in a humidified $5 \%$ carbon dioxide atmosphere in the presence of SJG-136 $\left(10^{-10}\right.$ $\left.10^{-7} \mathrm{M}\right)$, fludarabine $\left(10^{-7}-10^{-5} \mathrm{M}\right)$ or both drugs in combination at fixed molar ratio of $1: 100$ (SJG-136: fludarabine). The optimal ratio for synergy between the two agents was determined experimentally (data not shown). In addition, control cultures were carried out to which no drug was added. All experiments were performed in duplicate.

\section{Single-cell gel electrophoresis assay}

The level of DNA ICL was determined using a modified single-cell gel electrophoresis (comet) assay (Hartley et al, 1999; Spanswick et al, 2002). Each sample was split into two for the separate analysis of single-strand breaks and DNA ICL. The sample for crosslink analysis was irradiated $\left(12.5 \mathrm{~Gy}, 2.35 \mathrm{~Gy} \mathrm{~min}^{-1}\right)$ immediately before analysis to deliver a fixed number of random DNAstrand breaks. After embedding cells in $1 \%$ agarose the cells were lysed for $1 \mathrm{~h}$ in lysis buffer and then washed for $1 \mathrm{~h}$ in distilled water. Slides were then incubated in alkali buffer for $45 \mathrm{~min}$ followed by electrophoresis in the same buffer for $25 \mathrm{~min}$.

After drying, the slides were stained with propidium iodide for $30 \mathrm{~min}$ then rinsed in distilled water. Images were captured using an online CCD camera and analysed using Komet Analysis software (Kinetic Imaging, Liverpool, UK). For each duplicate slide, 25 cells were analysed. The tail moment for each image was calculated using the Komet Analysis software as the product of the percentage DNA in the comet tail and the distance between the

Table I Clinical characteristics of the CLL patients in this study

\begin{tabular}{ll}
\hline No. of patients & 40 \\
Mean age (years) & 64 \\
Sex (male/female) & $25 / 15$ \\
Binet stage $(\mathrm{A} / \mathrm{B} / \mathrm{C})$ & $13 / 9 / 18$ \\
Previous treatment (untreated/treated) & $24 / 16$ \\
Clinical resistance to fludarabine (resistant/sensitive) & $9 / 16$ \\
$V_{H}$ gene mutation (mutated/unmutated) & $18 / 22$ \\
CD38 expression $(<30 \% / \geqslant 30 \%)$ & $17 / 23$ \\
$Z$ ZAP-70 expression $(<20 \% / \geqslant 20 \%)$ & $15 / 25$ \\
\hline
\end{tabular}

$\mathrm{CLL}=$ chronic lymphocytic leukaemia. means of the head and tail distributions, based on the definition of Olive et al (1990). Crosslinking was expressed as the percentage decrease in tail moment compared to irradiated controls and was calculated using the formula

$$
\% \text { decrease in tail moment }=\left[1-\left(\frac{\mathrm{TMdi}-\mathrm{TMcu}}{\mathrm{TMci}-\mathrm{TMcu}}\right)\right] \times 100
$$

where TMdi is the tail moment of drug-treated irradiated sample, TMcu the tail moment of untreated, unirradiated control, and TMci the tail moment of untreated, irradiated.

In combination studies of SJG-136 and fludarabine, the following formula was used:

$$
\begin{aligned}
& \% \text { decrease in tail moment }= \\
& {\left[1-\left(\frac{(\mathrm{TMdi}-\mathrm{TMcu})}{(\mathrm{TMci}-\mathrm{TMcu})+(\mathrm{TM} d u-\mathrm{TMcu})}\right)\right] \times 100}
\end{aligned}
$$

where TMdu is the tail moment of drug-treated, unirradiated samples to take into account any extra strand breaks produced by fludarabine.

\section{Measurement of in vitro apoptosis}

Cultured cells were harvested by centrifugation. Apoptosis was assessed by dual-colour immunofluorescent flow cytometry as described previously (Vermes et al, 1997; Pepper et al, 2003).

\section{Assessment of synergy between SJG-136 and fludarabine}

Cells were incubated with various concentrations of fludarabine, SJG-136 and fludarabine in an optimised fixed-ratio combination with SJG- 136 for $48 \mathrm{~h}$ at $37^{\circ} \mathrm{C}$. Dose - response curves were plotted for each individual drug and drug combination, and the curves were analysed using the median effect method to determine the degree of synergy (Chou and Talalay, 1984). These experiments were conducted in two groups of samples, that is those derived from patients who had demonstrated either in vitro and/or clinical resistance to fludarabine (20 patients) and CLL samples that retained sensitivity to fludarabine ( 20 patients) to assess the drug interactions in each cohort.

\section{RT - PCR analysis of ERCC1 transcription}

Total RNA was extracted from $3 \times 10^{7}$ CLL lymphocytes using RNeasy spin columns according to the manufacturer's instructions (Qiagen, Crawley, UK). One microgram of total RNA was then reverse-transcribed using a Clontech first-strand cDNA synthesis kit (Clontech, Between Towns, Cowley, Oxford, UK). Subsequently, real-time PCR was performed using ERCC1-specific primers CCGCCAGCAAGGAAGAAATT (forward) and TTACGTCGCC AAATTCCCAG (reverse) and S14 (house-keeping gene) primers GGCAGACCGAGATGAACTCT (forward) and CCAGGTCCAGGG GTCTTGGT (reverse) in PCR buffer containing $50 \mathrm{~nm}$ of each primer, $4.0 \mathrm{~mm} \mathrm{MgCl}_{2}$ cDNA template and FastStart SYBR Green I DNA master mix (Roche Diagnostics, Welwyn Garden City, Hertfordshire, UK) in a $10 \mu \mathrm{l}$ final volume. Each reaction was cycled 45 times consisting of a $10 \mathrm{~s}$ denaturation $\left(95^{\circ} \mathrm{C}\right), 5 \mathrm{~s}$ annealing $\left(60^{\circ} \mathrm{C}\right)$ and $20 \mathrm{~s}$ extension $\left(72^{\circ} \mathrm{C}\right)$. To confirm the amplification specificity the PCR products were subjected to melting-curve analysis. The S14:ERCC1 transcription ratio was calculated from the crossing points of each gene.

\section{Statistical analysis}

The data obtained in these experiments were subjected to the Kolmorgorov-Smirnov test of normality; all the data sets tested were shown to be Gaussian. Consequently, statistical analyses were performed using the paired Student's $t$-test. Correlation 
coefficients were calculated from least-squares linear regression plots, and drug sensitivity was evaluated using nonlinear regression and line of best-fit analysis of the sigmoidal dose-response curves. All statistical analyses were performed using Graphpad Prism 3.0 software (Graphpad Software Inc., San Diego, CA, USA). Synergy was assessed using Calcusyn software (Biosoft, Cambridge, UK).

\section{RESULTS}

\section{SJG-136-induced DNA ICL}

The level of DNA ICL was determined using a modification of the comet assay. Figure $1 \mathrm{~A}$ shows the mean ( \pm s.d.) percentage decrease in tail moment in CLL samples derived from 10 patients following exposure to 2 and $4 \mathrm{~nm}$ SJG-136 for $24 \mathrm{~h}$. A decrease in tail moment has previously been shown to reflect an increase in DNA ICL (Hartley et al, 1999). Therefore, SJG-136 was shown to be a potent ICL agent in all the CLL samples tested with a mean concentration of SJG-136 that caused a 50\% decrease in tail moment of approximately $2.6 \mathrm{nM}$. Importantly, the degree of DNA crosslinking was correlated with in vitro sensitivity to SJG-136 as determined by measurement of apoptosis using Annexin V (Figure 1B) suggesting that ICL formation was pivotal to the cytotoxic effects of SJG-136.

The addition of fludarabine at a fixed molar ratio of $1: 100$ (SJG136 : fludarabine) resulted in increased DNA crosslinking in the majority of samples tested (9/10) with an average increase of $32.8 \%$ at the $2 \mathrm{nM}$ concentration of SJG-136. The comparisons of DNA crosslinking at $2 \mathrm{nM}$ and $4 \mathrm{nM}$ SJG-136 \pm fludarabine are shown in Figure 1C. We also performed time-course experiments using both the comet assay and the Annexin V assay to establish the kinetics of the DNA crosslinking and apoptosis induction in primary CLL cells. In keeping with previous findings (Clingen et al, 2005), SJG136 rapidly induced DNA crosslinks and these were maximal after $24 \mathrm{~h}$ exposure (data not shown). Apoptosis was also induced in a time-dependent manner with clear evidence of enhanced apoptotic cell killing in the cultures exposed to the combination of SJG-136 and fludarabine (Figure 1D). By $12 \mathrm{~h}$ the combination was statistically more cytotoxic than SJG-136 alone $(P=0.04)$ and by $24 \mathrm{~h}$ this was even more marked $(P<0.0001)$. This enhanced cytotoxicity appeared greater than the summative apoptotic effects of the two agents alone suggesting a synergistic interaction.

\section{SJG-136 and fludarabine synergise in primary CLL cells}

The cytotoxic synergy between SJG-136 and fludarabine was assessed in all 40 samples. Figure $2 \mathrm{~A}$ shows the dose-response of CLL cells derived from one patient treated with various concentrations of SJG-136 and fludarabine as single agents and in combination. The combination of the two drugs was significantly more cytotoxic than either single agent. Furthermore, Figure 2B shows that the slopes of the median-effect plots for the single drugs and the combination were not parallel, suggesting that the two agents had different modes of action. Therefore, the equation for the conservative isobologram was used to calculate the combination index (CI) shown in the fraction-affected CI plot (Figure 2C). All 40 samples showed synergy between the two drugs at a fixed molar ratio of $1: 100$ (SJG-136: fludarabine) with a mean CI of $0.54 \pm 0.2$. Combination index values less than 1 denote synergy, with the smallest values representing the greatest degree of synergy. Although the degree of synergy was significantly diminished in patient samples defined as fludarabine resistant
A
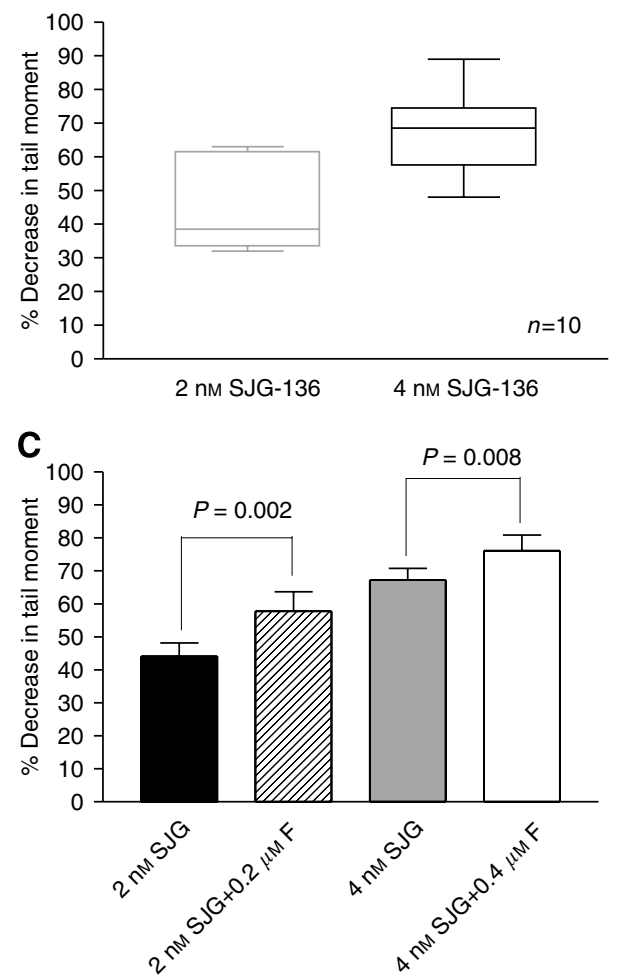

B
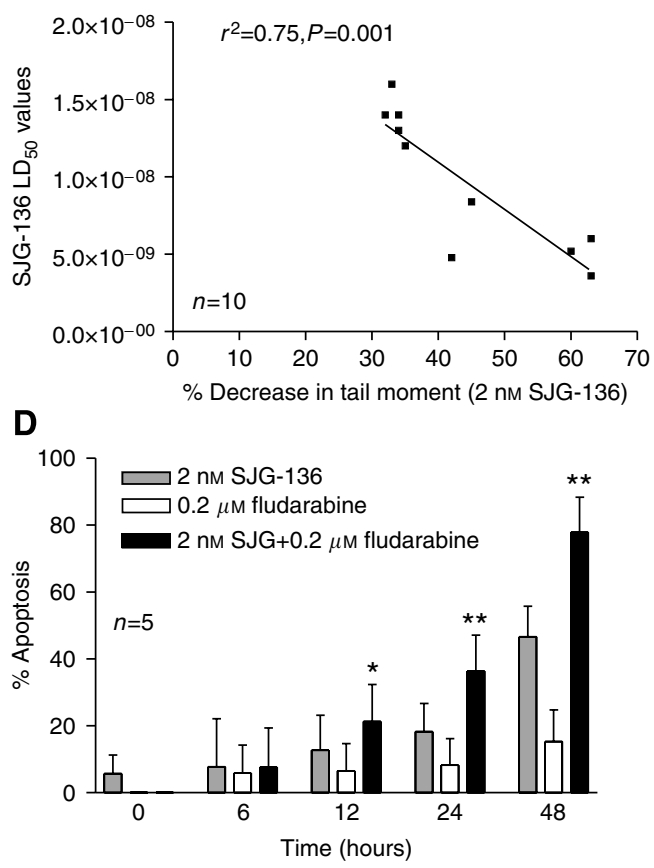

Figure I Percentage decrease in comet tail moment in CLL samples treated with SJG-136 \pm fludarabine for $24 \mathrm{~h}$. The decrease in tail moment reflects an increase in DNA ICL. (A) Ability of SIG-I36 to produce DNA ICL in a concentration-dependent manner in the samples tested. (B) The degree of DNA crosslinking correlated with the cytotoxic effects of SJG-I 36 in vitro. (C) Effect of the combination of SJG-I 36 and fludarabine resulting in increased DNA crosslinking. (D) Apoptosis was markedly enhanced in a time-dependent manner in samples treated with the combination of SJG-I36 and fludarabine when compared to either drug alone. The increase in apoptosis was evident after $12 \mathrm{~h}(* \mathrm{P}<0.05)$ and became more marked after 24 and $48 \mathrm{~h}(* * \mathrm{P}<0.000 \mathrm{I})$. 

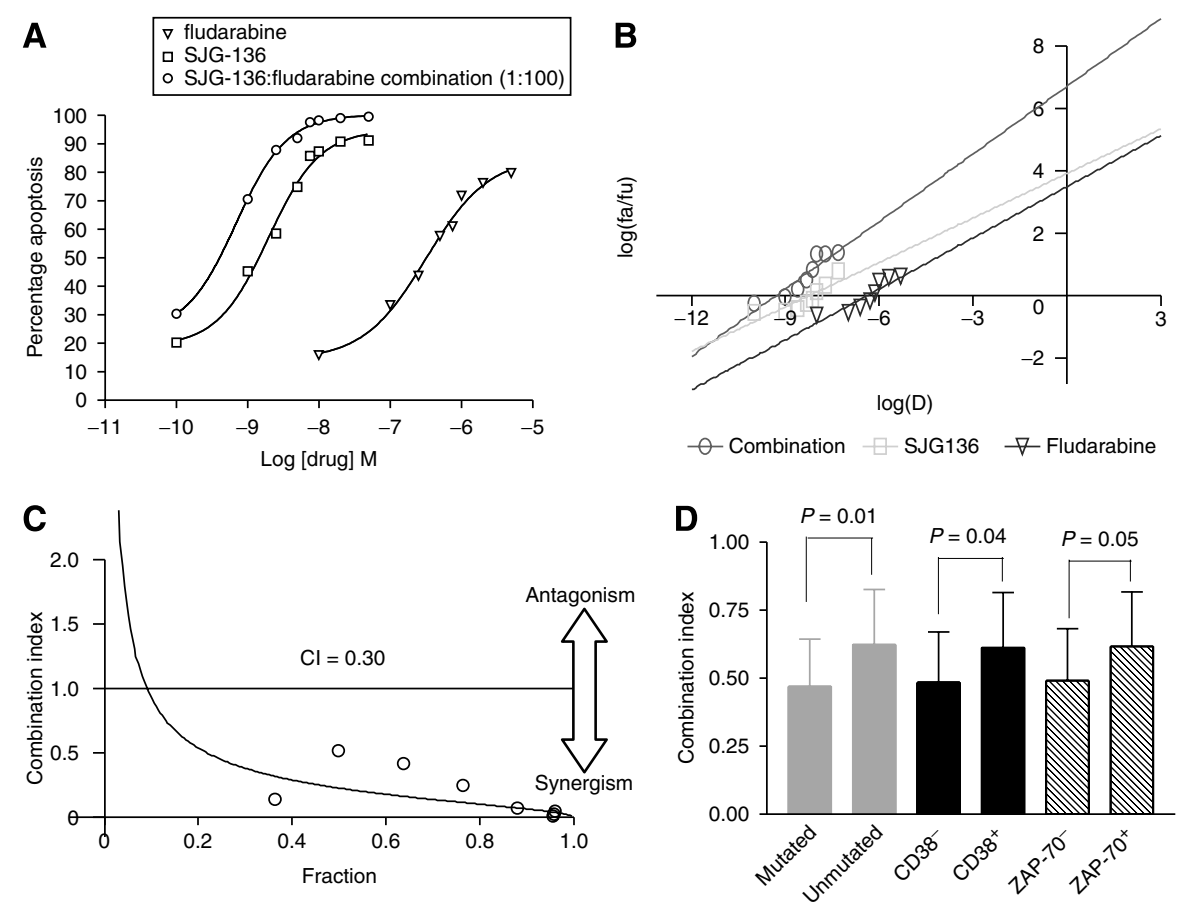

Figure 2 Synergistic cytotoxicity produced by SJG-I36 and fludarabine in primary CLL cells. (A) Dose-response curves were generated for CLL cells treated with SJG- 136 and/or fludarabine. The cytotoxicity was quantified using an Annexin V/propidium iodide assay. (B) Median-effect plot. The cells were treated for $48 \mathrm{~h}$ with SJG-I 36 (I.0, 2.5, 5.0, 7.5, 10.0, 20.0 or $50 \mathrm{~nm})$, fludarabine $(0.1,0.25,0.5,0.75,1.0,2.0$ or $5.0 \mu \mathrm{M})$, or both at a fixed molar ratio of I : 100. The median-effect plot was constructed using Calcusyn software where Fa is the fraction affected and Fu the fraction unaffected. The fractionaffected $\mathrm{Cl}$ plot was constructed by computer analysis of the data in $(\mathbf{B})$ using the conservative isobologram $(\mathbf{C})$. Combination index values of $<$ I occurred at a wide range of inhibition levels, indicating that synergy was produced by the combination. (D) Analysis of prognostic subsets revealed that there was diminished synergy in samples derived from patients with unmutated $V_{H}$ genes, high CD38 expression and high ZAP-70 expression. However, this is likely to be the result of a preponderance of these samples demonstrating in vitro or in vivo fludarabine resistance.

Table 2 Mean drug sensitivity data for the fludarabine sensitive and fludarabine resistant subsets of CLL samples in the study

\begin{tabular}{lccc}
\hline & $\begin{array}{c}\text { Mean } \\
\text { fludarabine } \\
\text { LD }_{\mathbf{5 0}}\end{array}$ & $\begin{array}{c}\text { Mean SJG-I36 } \\
\text { LD }_{\mathbf{5 0}}\end{array}$ & $\begin{array}{c}\text { Mean } \\
\mathbf{C l}\end{array}$ \\
\hline Fludarabine sensitive $(n=20)$ & $0.8 \mu \mathrm{M} \pm 0.5 \mu \mathrm{M}$ & $8.5 \mathrm{nM} \pm 3.0 \mathrm{nM}$ & $0.47 \pm 0.2$ \\
Fludarabine resistant $(n=20)$ & $1.7 \mu \mathrm{M} \pm 0.6 \mu \mathrm{M}$ & $10.2 \mathrm{nM} \pm 3.2 \mathrm{nM}$ & $0.62 \pm 0.3$ \\
$P$-value & $0.004 *$ & 0.13 & $0.03 *$ \\
\hline
\end{tabular}

$\mathrm{Cl}=$ combination index; $\mathrm{CLL}=$ chronic lymphocytic leukaemia. *Statistical significance at $P<0.05$ level.

when compared with fludarabine-sensitive samples $(P=0.03)$, synergy was still demonstrated in all these samples (mean $\mathrm{CI}=0.62 \pm 0.3$ ). The drug-sensitivity data for fludarabine-sensitive and fludarabine-resistant patient groups are summarised in Table 2. Analysis of the degree of synergy, as defined by $\mathrm{CI}$ values, in prognostically distinct subsets of CLL patients revealed that patients with unmutated $\mathrm{V}_{\mathrm{H}}$ genes, high CD38 expression and high ZAP-70 expression all showed significantly reduced synergy (Figure 2D). However, this observation is likely to be caused by a greater proportion of these subsets having either in vitro or in vivo fludarabine resistance.

\section{Inhibition of transcription of the excision repair enzyme ERCC1 by fludarabine}

The consistent demonstration of synergy between SJG-136 and fludarabine in this study led us to consider the possible mechanism that potentiated this effect. It has previously been demonstrated that fludarabine can inhibit DNA repair mechanisms and thereby synergise with cisplatin in the human chronic myeloid leukaemic K562 cell line (Yang et al, 1995; Li et al, 1997). More recently, Clingen et al (2005) reported that the XPF-ERCC1 endonuclease contributes to the repair of SJG-136-induced minor groove DNA ICL in Chinese hamster ovary (CHO) cells. Therefore, we hypothesised that fludarabine may suppress ERCC1 transcription and thereby enhance the cytotoxic effects of SJG-136. We first analysed serial dilutions of cDNA derived from CLL patient RNA to compare the efficiency of the PCR amplification reactions for ERCC1 and S14 (Figure 3A). It could be demonstrated in three independent experiments that both PCR ran with almost identical amplification efficiency, which is a prerequisite for using the change in crossing point $\left(\Delta C_{\mathrm{t}}\right)$ method for relative quantitation (Winer et al, 1999). To take into account the variation in total RNA introduced into each reaction, transcripts of $\$ 14$ were quantified as an endogenous RNA control, and each sample was normalised on the basis of its S14 content. In addition, the relative amounts of ERCC1 and S14 were also normalised to a control sample (untreated cells). There was interpatient variability in terms of the magnitude of change in ERCC1 transcription following exposure to both fludarabine and SJG-136 (Figure 3B). However, fludarabine suppressed ERCC1 transcription in the majority of samples (15/ $20)$ and SJG-136 caused an increase in ERCC1 transcription (20/20) when compared to controls. Analysis of all 20 patients studied (Figure 3C) confirmed that fludarabine significantly suppressed ERCC1 transcription $(P=0.04)$, SJG-136 significantly induced ERCC1 transcription $(P<0.0001)$ and the combination of fludarabine and SJG-136 resulted in a suppression of ERCC1 transcription when compared to SJG-136 alone $(P=0.001)$. 

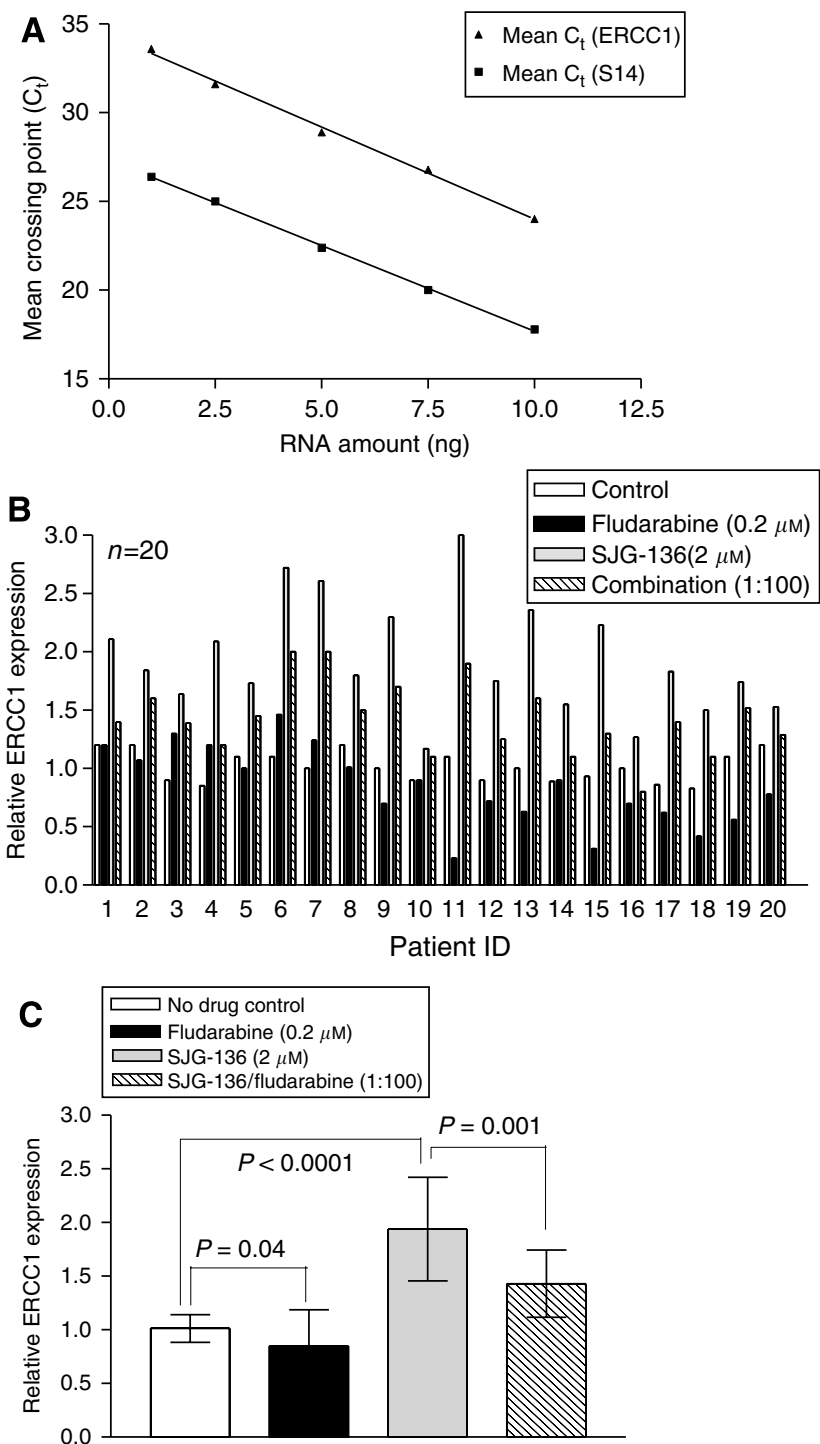

Figure 3 Real-time quantitative PCR for ERCC-I expression. Initial amount of cDNA in each reaction was plotted against the $C_{t}$ values for the house-keeping gene SI 4 and the target gene ERCC-I, and standard curves plotted. (A) The correlation coefficient for each curve was 0.99 suggesting that the amplification efficiencies for each reaction were similar. Data are representative of three independent experiments. (B) There was marked interpatient variability in ERCCl transcription following exposure to fludarabine, SJG-I36 or a combination of both agents. (C) The mean change in ERCCl transcription $( \pm)$ s.d. normalised to the control (no drug) sample for all 20 patients revealed that fludarabine suppressed ERCCI transcription both alone and in combination with SJG-136.

\section{Suppression of ERCC1 transcription correlates with the degree of synergy observed with SJG-136}

We next investigated whether fludarabine-mediated suppression of ERCC1 transcription was mechanistically relevant to the synergy observed between SJG-136 and fludarabine. To do this, we compared the percentage reduction in ERCC1 transcription induced by fludarabine in each patient sample with the degree of synergy between SJG-136 and fludarabine as measured by the CI. Figure $4 \mathrm{~A}$ shows the positive correlation between these two parameters suggesting a mechanistic link between increased suppression of ERCC1 transcription and enhanced synergy
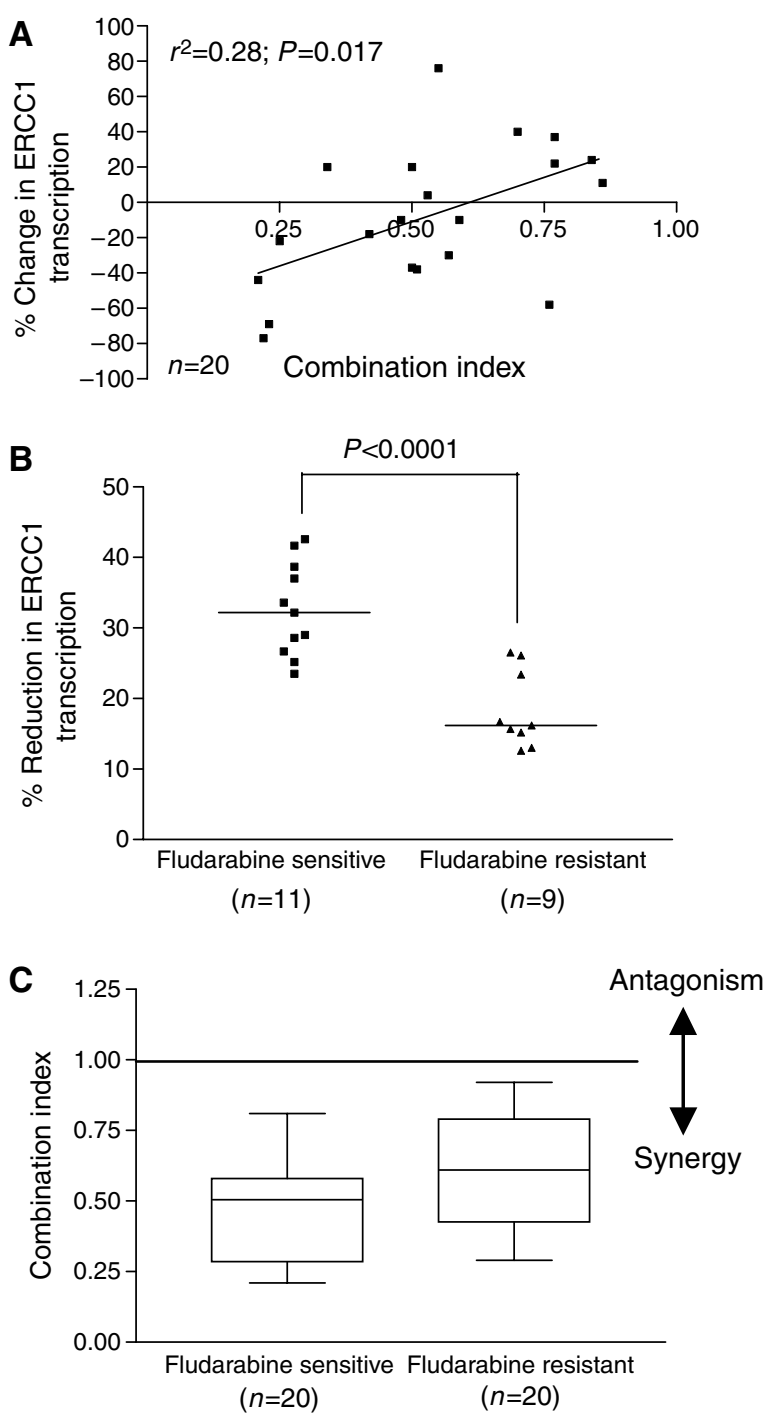

Figure 4 Relationship between change in $\mathrm{ERCCI}$ transcription and the degree of synergy between SJG-136 and fludarabine. (A) The percentage change in ERCCI transcription induced by fludarabine correlated with the degree of synergy observed between SJG-I 36 and fludarabine as measured by $\mathrm{Cl}$. (B) The fludarabine-sensitive samples showed a significant increased ability to suppress ERCCI transcription when compared with the fludarabine-resistant subset $(P<0.000 \mathrm{I})$. (C) This resulted in significantly diminished synergy in the fludarabine-resistant subset $(P=0.03)$.

between the two drugs. Further evidence for this association was derived from subset analysis of the fludarabine-sensitive and fludarabine-resistant patient groups. The diminished degree of synergy demonstrated in the fludarabine-resistant subset was strongly associated with the reduced capacity of fludarabine to suppress ERCC1 transcription (Figure 4B and C). However, the retention of synergy in these samples indicates that the mechanisms that underpin the synergistic interaction between fludarabine and SJG-136 are more complex than the transcriptional regulation of one excision repair enzyme.

\section{DISCUSSION}

In this study, we set out to establish whether fludarabine could enhance the DNA ICL capacity of SJG-136 in primary human CLL cells and thereby offer a rationale for its clinical use in 
combination with SJG-136. Using a modified comet assay to measure DNA crosslinking, we demonstrated that SJG-136 is an efficient, concentration-dependent, ICL-inducing agent in primary CLL cells. Importantly, the level of ICL correlated with sensitivity to SJG-136, suggesting a causal link between this critical lesion and cytotoxicity. Time course analysis revealed that the percentage of SJG-136-induced ICL was similar at both 24 and $48 \mathrm{~h}$ (data not shown). These results are in accord with those described by Hartley et al (2004), who showed that SJG-136-induced DNA ICL persisted both in vitro and in vivo when compared to those produced by conventional major groove binding drugs inferring that the kinetics of the repair of SJG-136-induced DNA crosslinking are very slow. However, co-incubation of SJG-136 and fludarabine resulted in an enhanced DNA crosslinking profile in the majority of the CLL cell samples tested (9/10), indicating that the combination may be more cytotoxic than SJG-136 alone.

Consequently, the next question that we wanted to address was to establish whether fludarabine could synergise with SJG-136 to induce more effective cell killing in primary human CLL cells. All of the samples tested $(n=40)$ at the experimentally optimised $1: 100$ fixed molar ratio (SJG-136:fludarabine) demonstrated a high level of synergy (mean $\mathrm{CI}=0.54 \pm 0.2$ ). Importantly, this synergistic relationship, albeit diminished, was retained even in samples derived from patients with either in vitro or in vivo fludarabine resistance (mean $\mathrm{CI}=0.62 \pm 0.3$ ). The fludarabineresistant subset was characterised by a predominance of samples derived from patients with poor prognostic markers; unmutated $\mathrm{V}_{\mathrm{H}}$ genes (16/20), high CD38 expression (14/20) and high ZAP-70 expression (12/20). As a result, subset analysis of these groups revealed statistically diminished synergy between SJG-136 and fludarabine in all of these poor prognostic subsets. However, synergy was retained in these poor risk groups, suggesting that the combination of SJG-136 and fludarabine may be particularly useful in these patients as well as in the salvage of fludarabine-resistant disease.

Previous work has shown that fludarabine can inhibit the repair of cisplatin- and oxaliplatin-induced DNA crosslinking (Yang et al, 1995; Li et al, 1997; Moufarij et al, 2006). Importantly, this appears to be more marked in CLL cells when compared to normal

\section{REFERENCES}

Alley MC, Hollingshead MG, Pacula-Cox CM, Waud WR, Hartley JA, Howard PW, Gregson SJ, Thurston DE, Sausville EA (2004) SJG-136 (NSC 694501), a novel rationally designed DNA minor groove interstrand cross-linking agent with potent and broad spectrum antitumor activity: part 2: efficacy evaluations. Cancer Res 64: $6700-6706$

Binet JL, Auquier A, Dighiero G, Chastang C, Piguet H, Goasguen J, Vaugier G, Potron G, Colona P, Oberling F, Thomas M, Tchernia G Jacquillat C, Boivin P, Lesty C, Duault MT, Monconduit M, Belabbes S, Gremy F (1981) A new prognostic classification of chronic lymphocytic leukemia derived from a multivariate survival analysis. Cancer 48: $198-206$

Bosch F, Montserrat E (2002) Refining prognostic factors in chronic lymphocytic leukemia. Rev Clin Exp Hematol 6: 335-349

Byrd JC, Murphy T, Howard RS, Lucas MS, Goodrich A, Park K, Pearson M, Waselenko JK, Ling G, Grever MR, Grillo-Lopez AJ, Rosenberg J, Kunkel L, Flinn IW (2001) Rituximab using a thrice weekly dosing schedule in B-cell chronic lymphocytic leukemia and small lymphocytic lymphoma demonstrates clinical activity and acceptable toxicity. J Clin Oncol 19: 2153-2164

Byrd JC, Peterson BL, Morrison VA, Park K, Jacobson R, Hoke E, Vardiman JW, Rai K, Schiffer CA, Larson RA (2003) Randomized phase 2 study of fludarabine with concurrent versus sequential treatment with rituximab in symptomatic, untreated patients with B-cell chronic lymphocytic leukemia: results from Cancer and Leukemia Group B 9712 (CALGB 9712). Blood 101: 6-14 lymphocytes (Moufarij et al, 2006). In addition, Clingen et al (2005) demonstrated that sensitivity to SJG-136 was dependent to some extent on ERCC1 expression in CHO cells. To determine whether ERCC1 was induced by SJG-136 in primary CLL cells, we performed real-time RT-PCR on RNA extracted from CLL cells exposed to SJG-136, fludarabine and both drugs in combination. All of the samples tested (20/20) showed a marked increase in ERCC1 transcription in response to SJG-136 when compared to untreated control samples. In contrast, fludarabine suppressed ERCC 1 transcription as a single agent in the majority of samples $(15 / 20)$ and inhibited SJG-136-induced ERCC1 transcription when used in combination (20/20). Importantly, the ability of fludarabine to suppress ERCC1 transcription when used in combination with SJG-136 correlated with the degree of synergy observed between the two drugs $\left(r^{2}=0.28 ; P=0.017\right)$. This indicates that the inhibition of ERCC1 is a key mechanistic component of the synergy between the two drugs. However, samples that showed diminished fludarabine-mediated suppression of ERCC1 still retained cytotoxic synergy between the two agents pointing to additional mechanisms that contribute to their synergistic interaction. Taken together, our data demonstrate a clear relationship between SJG-136-induced DNA ICL and cytotoxicity in primary CLL cells and offer a mechanism-based rationale for the increased DNA ICL observed in samples treated with the combination of SJG-136 and fludarabine compared to SJG-136 alone. The retention of cytotoxic synergy in samples derived from patients with either in vitro or in vivo resistance to fludarabine provides a clear indication for the use of the combination of SJG-136 and fludarabine in the treatment of this group of patients.

\section{ACKNOWLEDGEMENTS}

We thank Spirogen Ltd and Ipsen Ltd for permission for these studies to be carried out. We are also grateful to Dr Robert Schultz of the NCI (USA) for supplying the sample of SJG-136 used in these experiments (Batch Number 694501-Z/5/0) and to Victoria Spanswick and Janet Hartley for their input into the comet assay.
Chou TC, Talalay P (1984) Quantitative analysis of dose-effect relationships: the combined effects of multiple drugs or enzyme inhibitors. $A d v$ Enzyme Regul 22: 27-55

Clingen PH, De SI, McHugh PJ, Ghadessy FJ, Tilby MJ, Thurston DE, Hartley JA (2005) The XPF-ERCC1 endonuclease and homologous recombination contribute to the repair of minor groove DNA interstrand crosslinks in mammalian cells produced by the pyrrolo[2,1-c] $[1,4]$ benzodiazepine dimer SJG-136. Nucleic Acids Res 33: 3283-3291

Gregson SJ, Howard PW, Hartley JA, Brooks NA, Adams LJ, Jenkins TC, Kelland LR, Thurston DE (2001) Design, synthesis, and evaluation of a novel pyrrolobenzodiazepine DNA-interactive agent with highly efficient cross-linking ability and potent cytotoxicity. J Med Chem 44: 737-748

Hartley JA, Spanswick VJ, Brooks N, Clingen PH, McHugh PJ, Hochhauser D, Pedley RB, Kelland LR, Alley MC, Schultz R, Hollingshead MG, Schweikart KM, Tomaszewski JE, Sausville EA, Gregson SJ, Howard PW, Thurston DE (2004) SJG-136 (NSC 694501), a novel rationally designed DNA minor groove interstrand cross-linking agent with potent and broad spectrum antitumor activity: part 1: cellular pharmacology, in vitro and initial in vivo antitumor activity. Cancer Res 64: 6693-6699

Hartley JM, Spanswick VJ, Gander M, Giacomini G, Whelan J, Souhami RL, Hartley JA (1999) Measurement of DNA cross-linking in patients on ifosfamide therapy using the single cell gel electrophoresis (comet) assay. Clin Cancer Res 5: 507-512

Kay NE (2006) Purine analogue-based chemotherapy regimens for patients with previously untreated B-chronic lymphocytic leukemia. Semin Hematol 43: S50-S54 
Keating MJ, Flinn I, Jain V, Binet JL, Hillmen P, Byrd J, Albitar M, Brettman L, Santabarbara P, Wacker B, Rai KR (2002) Therapeutic role of alemtuzumab (Campath-1H) in patients who have failed fludarabine: results of a large International Study. Blood 99: 3554-3561

Lee JS, Dixon DO, Kantarjian HM, Keating MJ, Talpaz M (1987) Prognosis of chronic lymphocytic leukemia: a multivariate regression analysis of 325 untreated patients. Blood 69: 929-936

Li L, Keating MJ, Plunkett W, Yang LY (1997) Fludarabine-mediated repair inhibition of cisplatin-induced DNA lesions in human chronic myelogenous leukemia-blast crisis K562 cells: induction of synergistic cytotoxicity independent of reversal of apoptosis resistance. Mol Pharmacol 52: $798-806$

Moufarij MA, Sampath D, Keating MJ, Plunkett W (2006) Fludarabine increases oxaliplatin cytotoxicity in normal and chronic lymphocytic leukemia lymphocytes by suppressing interstrand DNA crosslink removal. Blood 108: 4187-4193

Olive PL, Banath JP, Durand RE (1990) Heterogeneity in radiation-induced DNA damage and repair in tumor and normal cells measured using the 'comet' assay. Radiat Res 122: 86-94

Pepper C, Thomas A, Hoy T, Milligan D, Bentley P, Fegan C (2003) The vitamin D3 analog EB1089 induces apoptosis via a p53-independent mechanism involving p38 MAP kinase activation and suppression of ERK activity in B-cell chronic lymphocytic leukemia cells in vitro. Blood 101: $2454-2460$

Pepper CJ, Hambly RM, Fegan CD, Delavault P, Thurston DE (2004) The novel sequence-specific DNA cross-linking agent SJG-136 (NSC 694501) has potent and selective in vitro cytotoxicity in human B-cell chronic lymphocytic leukemia cells with evidence of a p53-independent mechanism of cell kill. Cancer Res 64: 6750-6755

Spanswick VJ, Craddock C, Sekhar M, Mahendra P, Shankaranarayana P, Hughes RG, Hochhauser D, Hartley JA (2002) Repair of DNA interstrand crosslinks as a mechanism of clinical resistance to melphalan in multiple myeloma. Blood 100: 224-229

Sunters A, Springer CJ, Bagshawe KD, Souhami RL, Hartley JA (1992) The cytotoxicity, DNA crosslinking ability and DNA sequence selectivity of the aniline mustards melphalan, chlorambucil and 4-[bis(2-chloroethyl)amino] benzoic acid. Biochem Pharmacol 44: $59-64$

Vermes I, Haanen C, Richel DJ, Schaafsma MR, Kalsbeek-Batenburg E, Reutelingsperger CP (1997) Apoptosis and secondary necrosis of lymphocytes in culture. Acta Haematol 98: 8-13

Winer J, Jung CK, Shackel I, Williams PM (1999) Development and validation of real-time quantitative reverse transcriptase-polymerase chain reaction for monitoring gene expression in cardiac myocytes in vitro. Anal Biochem 270: $41-49$

Yang LY, Li L, Keating MJ, Plunkett W (1995) Arabinosyl-2-fluoroadenine augments cisplatin cytotoxicity and inhibits cisplatin-DNA cross-link repair. Mol Pharmacol 47: 1072-1079

Zhu Q, Tan DC, Samuel M, Chan ES, Linn YC (2004) Fludarabine in comparison to alkylator-based regimen as induction therapy for chronic lymphocytic leukemia: a systematic review and meta-analysis. Leuk Lymphoma 45: 2239-2245 